06

\title{
Генерация второй гармоники-суммарной частоты в тонком сферическом слое. І. Аналитическое решение
}

\author{
(C) В.Н. Капшай, А.И. Толкачёв, А.А. Шамына \\ Гомельский государственный университет им. Ф. Скорины, \\ 246019 Гомель, Республика Беларусь \\ e-mail: kapshai@rambler.ru, anton.talkachov@gmail.com, anton.shamyna@gmail.com
}

Поступила в редакцию 06.06.2021 г.

В окончательной редакции 23.08.2021 г.

Принята к публикации 02.09.2021 г.

В рамках модели Рэлея-Ганса-Дебая решена задача об одновременной генерации второй гармоники и суммарной частоты в тонком сферическом слое при его облучении двумя когерентными плоскими электромагнитными волнами с эллиптическими поляризациями и одинаковыми частотами. Тензор диэлектрической восприимчивости второго порядка выбран в виде, содержащем четыре независимые компоненты, в том числе одну киральную. Показаны принципиальные отличия в постановке и решении задачи о нелинейной генерации второго порядка при падении нескольких когерентных электромагнитных волн от задачи о генерации суммарной частоты в предельном случае равенства частот падающих волн. Определены сочетания параметров, при которых пространственное распределение генерируемого излучения при падении двух плоских волн совпадает с распределением излучения при генерации второй гармоники одной плоской волной. Рассмотрены предельные случаи полученного решения: малый и большой радиусы сферической частицы. При этом определены вклады в генерируемое излучение киральных и некиральных коэффициентов анизотропии.

Ключевые слова: генерация второй гармоники, генерация суммарной частоты, одинаковые частоты, диэлектрическая сферическая частица, тензор нелинейной восприимчивости.

DOI: $10.21883 / \mathrm{OS} .2021 .12 .51742 .2385-21$

\section{Введение}

В последние десятилетия для изучения поверхностей малых диэлектрических частиц используются нелинейные оптические явления, такие как генерация второй гармоники $[1,2]$ и генерация суммарной частоты $[3,4]$. Коллоидные растворы с такими частицами обычно облучают одним лазером и измеряют всевозможные характеристики индуцированных полей (например, плотность мощности, поляризацию и т.д.). В дипольном приближении нелинейные оптические эффекты второго порядка запрещены в центрально симметричных средах. На поверхности частиц условие центральной симметричности нарушается [4], что позволяет экспериментально обнаруживать генерируемое излучение. Генерируемые при этом поля значительно слабее исходных (от источников), что усложняет задачу их измерения. Это приводит к необходимости обработки частиц оптически нелинейным веществом $[1,2,5]$, таким как малахитовый зеленый, и проведения дополнительных измерений с повышением точности.

В опубликованных теоретических работах и при проведении экспериментальных исследований, связанных с генерацией второй гармоники, в качестве источника первичного излучения используется один лазер [5-8]. При этом варьируемыми параметрами, характеризующими источник и влияющими на параметры генерируемого излучения, являются только частота падающей волны, ее интенсивность и эллиптичность.
В настоящей работе мы предлагаем для изучения нелинейной генерации использовать два (или более) когерентных источника одинаковой частоты. В случае двух когерентных источников одинаковой частоты их суммарная частота совпадает, очевидно, с частотой второй гармоники. Будем говорить об этом явлении как о генерации второй гармоники-суммарной частоты. Наряду с этим можно изменять большее число параметров, связанных с исходными волнами: их частоту, интенсивности, направления волновых векторов, эллиптичности и ориентации эллипсов поляризации.

Ранее в работе [9] использовалась методика разделения лазерного пучка на две части с последующим пересечением данных пучков под некоторым углом на исследуемом образце. Целью данной методики было определение толщины образца, в объеме которого происходила генерация второй гармоники. Авторы работы [9] использовали термин „генерация суммарной частоты пересекающихся пучков“ (crossed-beam frequency summation) для происходящего явления. Происходили независимо три явления: генерации второй гармоники каждой волной и генерация суммарной частоты. Однако пересекающиеся пучки не были созданы когерентными, что подтверждалось путем изменения оптического пути одного из лучей, из-за чего наблюдаемая интенсивность являлась суммой интенсивностей вышеупомянутых явлений.

В ходе аналитического описания генерации второй гармоники-суммарной частоты при облучении частицы 
двумя когерентными источниками необходимо учитывать интерференцию исходных волн. При этом вектор поляризации среды содержит слагаемые, отвечающие за генерацию второй гармоники от каждого из источников в отдельности, и интерференционные слагаемые, которые рассматриваются в работах о генерации суммарной частоты.

\section{Аналитическое решение}

\section{Нелинейная часть вектора поляризации}

В настоящей работе генерируемые поля второй гармоники-суммарной частоты рассчитываются в рамках нелинейной модели Рэлея-Ганса-Дебая. В этой модели предполагается, что рассеяние вносит малый вклад в результирующее поле первой гармоники, и этим вкладом можно пренебречь. Таким образом, исходные волны поляризуют нелинейный слой на поверхности диэлектрической частицы, распространяясь без преломления и поглощения. Аналогично и генерируемые электромагнитные волны не испытывают рассеяния на поверхностях, разделяющих объем частицы, тонкий слой оптически нелинейного вещества и окружающую среду. Условиями применимости указанной модели являются близость относительного показателя преломления к единице и ограничение на размер (радиус) частицы, что выражается в виде следующих численных критериев:

$$
\left|\frac{n_{p}}{n_{m}}-1\right| \ll 1, \quad 4 \pi \frac{a}{\lambda}\left|\frac{n_{p}}{n_{m}}-1\right| \ll 1,
$$

где $n_{p}$ и $n_{m}$ - показатели преломления сферической частицы и окружающей среды соответственно, $a$ радиус частицы, $\lambda$ - длина волны падающего излучения в среде, окружающей частицу.

При облучении диэлектрической частицы двумя электромагнитными волнами в оптически нелинейном слое на ее поверхности происходят сразу три явления: генерация второй гармоники первой волной, генерация второй гармоники второй волной и генерация суммарной частоты двумя падающими волнами. Причем длина волны излучения суммарной частоты совпадает с длиной волны излучения второй гармоники, генерируемого каждой падающей волной. Это объясняется тем, что частоты падающих электромагнитных волн совпадают. В дипольной модели генерация поля второй гармоникисуммарной частоты обусловлена нелинейной частью вектора поляризации среды, компоненты которого с учетом вышесказанного можно записать в виде

$$
P_{i}^{(2)}=\chi_{i j k}^{(2)} E_{j}^{(1)} E_{k}^{(1)}+\chi_{i j k}^{(2)} E_{j}^{(2)} E_{k}^{(2)}+\chi_{\mathrm{SFG}, i j k}^{(2)} E_{j}^{(1)} E_{k}^{(2)},
$$

где $\chi_{i j k}^{(2)}$ и $\chi_{\mathrm{SFG}, i j k}^{(2)}-$ тензоры нелинейной диэлектрической восприимчивости второго порядка для генерации второй гармоники [8] и генерации суммарной частоты $[3,10]$ соответственно, $E_{j}^{(\alpha)}, E_{k}^{(\beta)}-$ компоненты векторов напряженности электрических полей падающих волн. Здесь и далее подразумевается правило суммирования по двум повторяющимся латинским индексам, а греческие индексы $\alpha$ и $\beta$ обозначают только принадлежность величины, соответствующей падающей волне, и принимают значения 1 или 2. Первое и второе слагаемые отвечают за генерацию второй гармоники первой и второй волной соответственно, а третье слагаемое отвечает за генерацию суммарной частоты двумя падающими волнами.

Отметим, что для первого и второго слагаемых в формуле (2) тензор нелинейной диэлектрической восприимчивости выбран одинаковым, поскольку обе падающих волны в нашей задаче имеют одинаковую частоту. В третьем слагаемом благодаря равенству частот падающих волн тензор $\chi_{\mathrm{SFG}, i j k}^{(2)}$ должен обладать симметрией относительно перестановки последних двух индексов. При рассмотрении явления генерации суммарной частоты $[3,10]$ условие перестановочной симметрии на тензор $\chi_{\mathrm{SFG}, i j k}^{(2)}$ не накладывается, ввиду чего в указанных работах рассматривается более общий вид данного тензора.

С другой стороны, нелинейную часть поляризации $P_{i}^{(2)}$ в любой точке поверхностного слоя можно рассмотреть, как генерацию второй гармоники. В этом случае излучение удвоенной частоты генерируется исходным электрическим полем, являющимся суперпозицией электрических полей падающих волн, т. е. здесь необходимо учитывать интерференцию падающих волн:

$$
\begin{gathered}
P_{i}^{(2)}=\chi_{i j k}^{(2)}\left(\mathbf{E}^{(1)}+\mathbf{E}^{(2)}\right)_{j}\left(\mathbf{E}^{(1)}+\mathbf{E}^{(2)}\right)_{k} \\
=\chi_{i j k}^{(2)} \sum_{\alpha=1}^{2} \sum_{\beta=1}^{2} E_{j}^{(\alpha)} E_{k}^{(\beta)} .
\end{gathered}
$$

Формулы (2) и (3) должны давать одинаковый результат, поскольку они описывают одно и то же явление генерации второй гармоники-суммарной частоты. Это возможно только в том случае, когда выполняется равенство

$$
\chi_{\mathrm{SFG}, i j k}^{(2)}=2 \chi_{i j k}^{(2)},
$$

т.е. каждая из компонент тензора нелинейной диэлектрической восприимчивости для генерации суммарной частоты в два раза больше соответствующих компонент для генерации второй гармоники. Далее будем работать только с компонентами тензора $\chi_{i j k}^{(2)}$, тогда компоненты тензора $\chi_{\mathrm{SFG}, i j k}^{(2)}$ при необходимости можно легко найти по формуле (4).

Тензор третьего ранга в самом общем виде содержит 27 компонент. Подчинение тензора $\chi_{i j k}^{(2)}$ свойствам симметрии при поворотах (группа $C_{\infty}$ по Шенфлису) и инверсии оставляют независимыми 7 компонент. Такой тензор используется для описания генерации суммарной частоты $[3,10]$, при этом имеются 3 киральных слагаемых. Дополнительное условие перестановочной симметрии $\chi_{i j k}^{(2)}$ по двум последним индексам, которое необ- 
ходимо накладывать как при рассмотрении генерации второй гармоники, так и при описании генерации второй гармоники-суммарной частоты, приводит к тензору $\chi_{i j k}^{(2)}$ с четырьмя независимыми компонентами [8] в виде

$$
\begin{aligned}
\chi_{i j k}^{(2)}= & \chi_{1}^{(2)} n_{i} n_{j} n_{k}+\chi_{2}^{(2)} n_{i} \delta_{j k}+\chi_{3}^{(2)}\left(n_{j} \delta_{i k}+n_{k} \delta_{i j}\right) \\
& +\chi_{4}^{(2)} n_{m}\left(n_{k} \varepsilon_{i j m}+n_{j} \varepsilon_{i k m}\right)
\end{aligned}
$$

Здесь $n_{i}-$ компоненты вектора нормали $\mathbf{n}$ к поверхности, $\delta_{i j}$ - дельта-символ Кронекера, $\varepsilon_{i j k}-$ символ Леви-Чивита, $\chi_{1-4}^{(2)}$ - значения независимых компонент тензора диэлектрической восприимчивости, последний коэффициент $\chi_{4}^{(2)}$ в $(5)$ называют киральным. Он фактически является псевдоскаляром, так как $\varepsilon_{i j k}-$ псевдотензор. Коэффициенты $\chi_{1-4}^{(2)}$ связаны с широко используемыми в литературе [5,7] независимыми компонентами тензора диэлектрической восприимчивости $\chi_{\perp \perp \perp}^{(2)}, \chi_{\perp\|\| \|}^{(2)}, \chi_{\|\perp\|}^{(2)}, \chi_{\|\| \perp}^{(2)}$ следующим образом:

$$
\begin{gathered}
\chi_{1}^{(2)}=\chi_{\perp \perp \perp}^{(2)}-\chi_{\perp\|\|}^{(2)}-2 \chi_{\|\perp\|}^{(2)}, \quad \chi_{2}^{(2)}=\chi_{\perp\|\|}^{(2)}=\chi_{\perp\left\|^{\prime}\right\|^{\prime}}^{(2)} \\
\chi_{3}^{(2)}=\chi_{\|\perp\|}^{(2)}=\chi_{\left\|^{\prime} \perp\right\|^{\prime}}^{(2)}=\chi_{\|\| \perp}^{(2)}=\chi_{\left\|^{\prime}\right\|^{\prime} \perp}^{(2)} \\
\chi_{4}^{(2)}=\chi_{\|\|^{\prime} \perp}^{(2)}=-\chi_{\left\|^{\prime}\right\| \perp}^{(2)}=\chi_{\|\perp\|^{\prime}}^{(2)}=-\chi_{\left\|^{\prime} \perp\right\|}^{(2)},
\end{gathered}
$$

где символы $\perp,\|,\|^{\prime}$ означают компоненты тензора вдоль осей, сонаправленных базисным векторам $\mathbf{n}_{\perp}=\mathbf{n}, \mathbf{n}_{\|}, \mathbf{n}_{\| \prime}$, где $\mathbf{n}_{\perp}$ перпендикулярен поверхности, а $\mathbf{n}_{\|}, \mathbf{n}_{\|^{\prime}}$ параллельны поверхности, причем $\mathbf{n}_{\perp}, \mathbf{n}_{\|}, \mathbf{n}_{\|}$ образуют правую тройку векторов (рис. 1).

\section{Решение задачи}

Электрические составляющие полей источников в выражении (3) зададим следующим образом:

$$
\mathbf{E}^{(\alpha)}(\mathbf{x}, t)=E_{\alpha} \mathbf{e}^{(\alpha)} \exp \left(i \mathbf{k}^{(\alpha)} \mathbf{x}-i \omega t\right)
$$

где $E_{\alpha}-$ комплексная амплитуда волны, $\mathbf{e}^{(\alpha)}-$ единичный комплексный вектор поляризации $\left(\mathbf{e}^{(1)^{*}} \mathbf{e}^{(1)}=\mathbf{e}^{(2)^{*}} \mathbf{e}^{(2)}=1\right), \quad \mathbf{k}^{(\alpha)}-$ волновой вектор, $\omega$ - циклическая частота.

Проводя рассуждения аналогично работе [8], векторный потенциал генерируемого поля второй гармоники-суммарной частоты представим в виде $\mathbf{A}(\mathbf{x}, t)=\mathbf{A}(\mathbf{x}) \exp (-i 2 \omega t), \quad$ где координатная часть определяется в методе функций Грина как

$$
\mathbf{A}(\mathbf{x})=-i k_{2 \omega} \sqrt{\frac{\mu_{2 \omega}}{\varepsilon_{2 \omega}}} \int_{V} \frac{e^{i k_{2 \omega}\left|\mathbf{x}-\mathbf{x}^{\prime}\right|}}{\left|\mathbf{x}-\mathbf{x}^{\prime}\right|} \mathbf{P}^{(2)}\left(\mathbf{x}^{\prime}\right) d^{3} \mathbf{x}^{\prime}
$$

Здесь $\varepsilon_{2 \omega}, \mu_{2 \omega}$ - диэлектрическая и магнитная проницаемости на частоте $2 \omega, k_{2 \omega}=\sqrt{\varepsilon_{2 \omega} \mu_{2 \omega}} 2 \omega / c$, интегрирование ведется по объему $V$, занятому веществом с нелинейными оптическими свойствами (по нелинейному слою). В приближении дальней зоны, когда

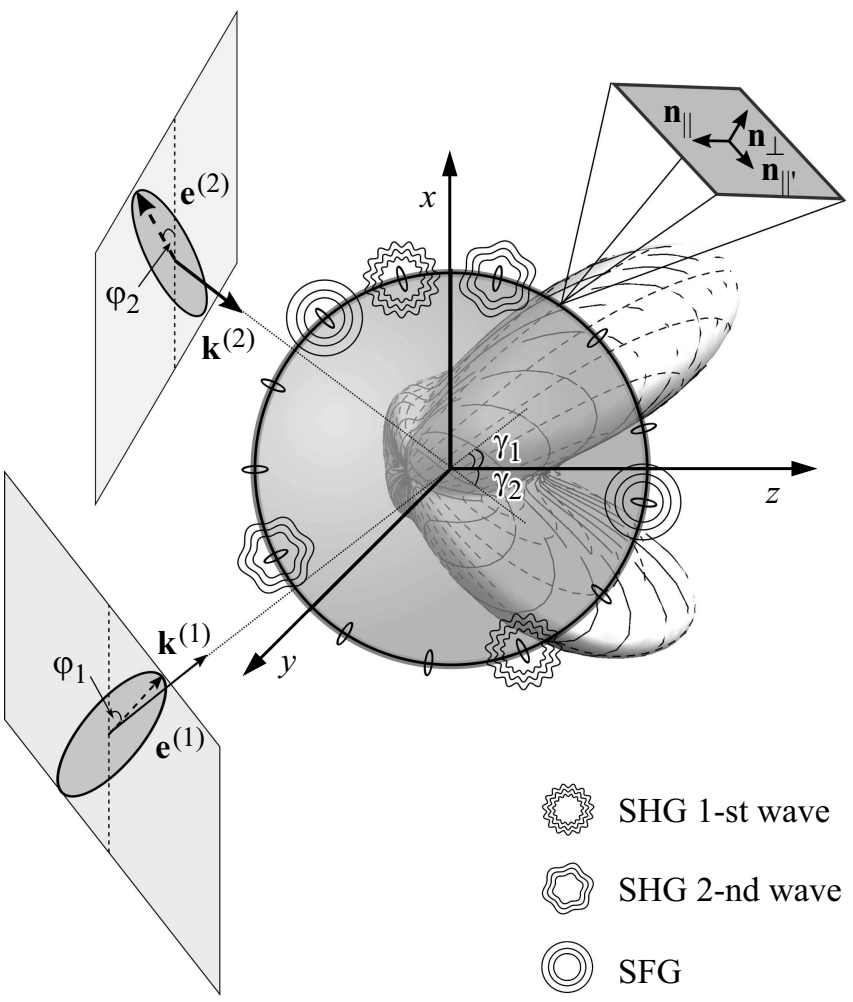

Рис. 1. Схема задачи о генерации второй гармоникисуммарной частоты.

$r=|\mathbf{x}| \gg\left|\mathbf{x}^{\prime}\right|$, получим

$\mathbf{A}(\mathbf{x})=-i k_{2 \omega} \sqrt{\frac{\mu_{2 \omega}}{\varepsilon_{2 \omega}}} \frac{e^{i k_{2 \omega} r}}{r} \int_{V} \exp \left(-i \mathbf{k}^{(2 \omega)} \mathbf{x}^{\prime}\right) \mathbf{P}^{(2)}\left(\mathbf{x}^{\prime}\right) d^{3} \mathbf{x}^{\prime}$,

где $\mathbf{k}^{(2 \omega)}=k_{2 \omega} \mathbf{e}_{r}-$ волновой вектор генерируемого поля. Тогда индукция магнитного и напряженность электрического полей запишутся в виде

$$
\begin{aligned}
\mathbf{B}^{(2 \omega)}(\mathbf{x})= & \operatorname{rot} \mathbf{A}(\mathbf{x}) \approx k_{2 \omega}^{2} \sqrt{\frac{\mu_{2 \omega}}{\varepsilon_{2 \omega}}} \frac{e^{i k_{2 \omega} r}}{r}\left[\mathbf{e}_{r}\right. \\
& \left.\times \int_{V} \exp \left(-i \mathbf{k}^{(2 \omega)} \mathbf{x}^{\prime}\right) \mathbf{P}^{(2)}\left(\mathbf{x}^{\prime}\right) d^{3} \mathbf{x}^{\prime}\right], \\
\mathbf{E}^{(2 \omega)}(\mathbf{x})= & \frac{i}{\left(\varepsilon_{2 \omega} \mu_{2 \omega} 2 \omega / c\right)} \operatorname{rot} \mathbf{B}^{(2 \omega)}(\mathbf{x}) \approx k_{2 \omega}^{2} \frac{e^{i k_{2 \omega} r}}{\varepsilon_{2 \omega} r} \\
& \times\left(1-\mathbf{e}_{r} \otimes \mathbf{e}_{r}\right) \int_{V} \exp \left(-i \mathbf{k}^{(2 \omega)} \mathbf{x}^{\prime}\right) \mathbf{P}^{(2)}\left(\mathbf{x}^{\prime}\right) d^{3} \mathbf{x}^{\prime},
\end{aligned}
$$

где символ $\otimes$ означает тензорное произведение векторов. Таким образом, зная магнитную индукцию, можно найти напряженность электрического поля. Можно, наоборот, выразить В через $\mathbf{E}$, пользуясь уравнениями Максвелла:

$$
\mathbf{B}(\mathbf{x})=\frac{-i c}{2 \omega} \operatorname{rot} \mathbf{E}(\mathbf{x})
$$


Поэтому дальнейшие рассуждения проводим для электрической составляющей поля.

Начала сферической и декартовой систем координат совместим с центром частицы (рис. 1), покрытой тонким нелинейным слоем толщины $d_{0} \ll a$. Подставляя напряженности (7) в (3), а результат в (11), получаем компоненты напряженности генерируемого электрического поля:

$$
\begin{aligned}
& E_{i}^{(2 \omega)}(\mathbf{x})=\mu_{2 \omega} \frac{(2 \omega)^{2}}{c^{2}} \frac{\exp \left(i k_{2 \omega} r\right)}{r}\left(\delta_{i m}-e_{r, i} e_{r, m}\right) \\
& \times \sum_{\alpha=1}^{2} \sum_{\beta=1}^{2} E_{\alpha} E_{\beta} e_{j}^{(\alpha)} e_{k}^{(\beta)} \int_{4 \pi} d \Omega_{\mathbf{x}^{\prime}} \int_{a}^{a+d_{0}} \exp \left(i \mathbf{q}^{(\alpha \beta)} \mathbf{x}^{\prime}\right) \\
& \times \chi_{m j k}^{(2)}\left(\mathbf{x}^{\prime}\right) r^{\prime 2} d r^{\prime},
\end{aligned}
$$

где $\mathbf{q}^{(\alpha \beta)}$ - векторы рассеяния (рис. 2, $\left.a, b\right)$, вычисляемые по формуле

$$
\mathbf{q}^{(\alpha \beta)}=\mathbf{k}^{(\alpha)}+\mathbf{k}^{(\beta)}-\mathbf{k}^{(2 \omega)}
$$

После интегрирования по радиусу остается интеграл по телесному углу при $\left|\mathbf{x}^{\prime}\right|=a$ :

$$
\begin{aligned}
& E_{i}^{(2 \omega)}(\mathbf{x})=\mu_{2 \omega} \frac{(2 \omega)^{2}}{c^{2}} \frac{\exp \left(i k_{2 \omega} r\right)}{r} d_{0} a^{2}\left(\delta_{i m}-e_{r, i} e_{r, m}\right) \\
& \times \sum_{\alpha=1}^{2} \sum_{\beta=1}^{2} E_{\alpha} E_{\beta} e_{j}^{(\alpha)} e_{k}^{(\beta)} \int_{4 \pi} \exp \left(i \mathbf{q}^{(\alpha \beta)} \mathbf{x}^{\prime}\right) \chi_{m j k}^{(2)}\left(\mathbf{x}^{\prime}\right) d \Omega_{\mathbf{x}^{\prime}} .
\end{aligned}
$$

Напряженность электрического поля второй гармоники-суммарной частоты в векторной форме может быть представлена в виде

$$
\begin{aligned}
& \mathbf{E}^{(2 \omega)}(\mathbf{x})=4 \pi \mu_{2 \omega} \frac{(2 \omega)^{2}}{c^{2}} \frac{\exp \left(i k_{2 \omega} r\right)}{r} d_{0} a^{2} \\
& \times\left(1-\mathbf{e}_{r} \otimes \mathbf{e}_{r}\right) \sum_{\alpha=1}^{2} \sum_{\beta=1}^{2} E_{\alpha} E_{\beta} \mathbf{f}^{(\alpha \beta)} .
\end{aligned}
$$

Здесь векторы $\mathbf{f}^{(\alpha \beta)}$ выражаются через тензоры эффективной нелинейной диэлектрической восприимчивости $\mathrm{X}_{i j k}^{(\alpha \beta)}$ формулами

$$
f_{i}^{(\alpha \beta)}=\mathrm{X}_{i j k}^{(\alpha \beta)} e_{j}^{(\alpha)} e_{k}^{(\beta)},
$$

а сами тензоры $\mathrm{X}_{i j k}^{(\alpha \beta)}$ выражаются в виде интегралов по телесному углу при $\left|\mathbf{x}^{\prime}\right|=a$ :

$$
\mathrm{X}_{i j k}^{(\alpha \beta)}=\frac{1}{4 \pi} \int_{4 \pi} \exp \left(i \mathbf{q}^{(\alpha \beta)} \mathbf{x}^{\prime}\right) \chi_{i j k}^{(2)}\left(\mathbf{x}^{\prime}\right) d \Omega_{\mathbf{x}^{\prime}} .
$$

Подставляя выражение (5) в (17), получаем интегралы следующих типов [10]:

$$
\int_{4 \pi} \exp \left(i a \mathbf{q}^{(\alpha \beta)} \mathbf{n}\right) n_{i} d \Omega_{\mathbf{x}^{\prime}}=4 \pi i v_{i}^{(\alpha \beta)} j_{1}\left(q^{(\alpha \beta)} a\right),
$$

$$
\begin{aligned}
& \int_{4 \pi} \exp \left(i a \mathbf{q}^{(\alpha \beta)} \mathbf{n}\right) n_{i} n_{j} d \Omega_{\mathbf{x}^{\prime}}=4 \pi\left[\delta _ { i j } \frac { 1 } { 3 } \left(j_{0}\left(q^{(\alpha \beta)} a\right)\right.\right. \\
& \left.\left.+j_{2}\left(q^{(\alpha \beta)} a\right)\right)-v_{i}^{(\alpha \beta)} v_{j}^{(\alpha \beta)} j_{2}\left(q^{(\alpha \beta)} a\right)\right] \\
& \int_{4 \pi} \exp \left(i a \mathbf{q}^{(\alpha \beta)} \mathbf{n}\right) n_{i} n_{j} n_{k} d \Omega_{\mathbf{x}^{\prime}}=4 \pi i\left[\frac { 1 } { 5 } \left(j_{1}\left(q^{(\alpha \beta)} a\right)\right.\right. \\
& \left.+j_{3}\left(q^{(\alpha \beta)} a\right)\right)\left(v_{i}^{(\alpha \beta)} \delta_{j k}+v_{j}^{(\alpha \beta)} \delta_{k i}\right. \\
& \left.\left.+v_{k}^{(\alpha \beta)} \delta_{i j}\right)-j_{3}\left(q^{(\alpha \beta)} a\right) v_{i}^{(\alpha \beta)} v_{j}^{(\alpha \beta)} v_{k}^{(\alpha \beta)}\right] .
\end{aligned}
$$

Здесь $\boldsymbol{v}^{(\alpha \beta)}$ - единичные векторы вдоль $\mathbf{q}^{(\alpha \beta)}$ (рис. 2, $a, b), q^{(\alpha \beta)}=\left|\mathbf{q}^{(\alpha \beta)}\right|, j_{m}\left(q^{(\alpha \beta)} a\right)-$ сферическая функция Бесселя порядка $m$. Тогда компоненты тензоров эффективной нелинейной диэлектрической восприимчивости записываются как

$$
\begin{aligned}
& \mathrm{X}_{i j k}^{(\alpha \beta)}=i \chi_{1}^{(2)}\left[-j_{3}\left(q^{(\alpha \beta)} a\right) v_{i}^{(\alpha \beta)} v_{j}^{(\alpha \beta)} v_{k}^{(\alpha \beta)}+\frac{1}{5}\left(j_{1}\left(q^{(\alpha \beta)} a\right)\right.\right. \\
& \left.\left.+j_{3}\left(q^{(\alpha \beta)} a\right)\right)\left(v_{i}^{(\alpha \beta)} \delta_{j k}+v_{j}^{(\alpha \beta)} \delta_{k i}+v_{k}^{(\alpha \beta)} \delta_{i j}\right)\right] \\
& +i j_{1}\left(q^{(\alpha \beta)} a\right)\left[\chi_{2}^{(2)} v_{i}^{(\alpha \beta)} \delta_{j k}+\chi_{3}^{(2)} v_{j}^{(\alpha \beta)} \delta_{i k}+\chi_{3}^{(2)} v_{k}^{(\alpha \beta)} \delta_{i j}\right] \\
& -\chi_{4}^{(2)} j_{2}\left(q^{(\alpha \beta)} a\right)\left(v_{k}^{(\alpha \beta)} v_{m}^{(\alpha \beta)} \varepsilon_{i j m}+v_{j}^{(\alpha \beta)} v_{m}^{(\alpha \beta)} \varepsilon_{i k m}\right)
\end{aligned}
$$

Переходя от компонентной записи (16) к векторной форме, для векторов $\mathbf{f}^{(\alpha \beta)}$ получим

$$
\begin{aligned}
& \mathbf{f}^{(\alpha \beta)}=i \chi_{1}^{(2)}\left(-j_{3}\left(q^{(\alpha \beta)} a\right) \boldsymbol{v}^{(\alpha \beta)}\left(\boldsymbol{v}^{(\alpha \beta)} \mathbf{e}^{(\alpha)}\right)\left(\boldsymbol{v}^{(\alpha \beta)} \mathbf{e}^{(\beta)}\right)\right. \\
& +\frac{1}{5}\left(j_{1}\left(q^{(\alpha \beta)} a\right)+j_{3}\left(q^{(\alpha \beta)} a\right)\right)\left(\boldsymbol{v}^{(\alpha \beta)}\left(\mathbf{e}^{(\alpha)} \mathbf{e}^{(\beta)}\right)\right. \\
& \left.\left.+\mathbf{e}^{(\beta)}\left(\boldsymbol{v}^{(\alpha \beta)} \mathbf{e}^{(\alpha)}\right)+\mathbf{e}^{(\alpha)}\left(\boldsymbol{v}^{(\alpha \beta)} \mathbf{e}^{(\beta)}\right)\right)\right)+i j_{1}\left(q^{(\alpha \beta)} a\right) \\
& \times\left(\chi_{2}^{(2)} \boldsymbol{v}^{(\alpha \beta)}\left(\mathbf{e}^{(\alpha)} \mathbf{e}^{(\beta)}\right)+\chi_{3}^{(2)} \mathbf{e}^{(\beta)}\left(\boldsymbol{v}^{(\alpha \beta)} \mathbf{e}^{(\alpha)}\right)\right. \\
& \left.+\chi_{3}^{(2)} \mathbf{e}^{(\alpha)}\left(\boldsymbol{v}^{(\alpha \beta)} \mathbf{e}^{(\beta)}\right)\right)-\chi_{4}^{(2)} j_{2}\left(q^{(\alpha \beta)} a\right) \\
& \times\left(\left[\mathbf{e}^{(\alpha)} \times \boldsymbol{v}^{(\alpha \beta)}\right]\left(\boldsymbol{v}^{(\alpha \beta)} \mathbf{e}^{(\beta)}\right)+\left[\mathbf{e}^{(\beta)} \times \boldsymbol{v}^{(\alpha \beta)}\right]\left(\boldsymbol{v}^{(\alpha \beta)} \mathbf{e}^{(\alpha)}\right)\right) .
\end{aligned}
$$

Аналитическое решение (15) с векторами $\mathbf{f}^{(\alpha \beta)}$, которые можно рассчитать по формуле (20), позволяет определить остальные компоненты и характеристики результирующего электромагнитного поля второй гармоникисуммарной частоты аналогично [10]. Для напряженности магнитного поля в дальней зоне верна формула, применимая к плоским волнам:

$$
\mathbf{H}^{(2 \omega)}(\mathbf{x})=\frac{n_{2 \omega}}{\mu_{2 \omega}}\left[\mathbf{e}_{r} \times \mathbf{E}^{(2 \omega)}(\mathbf{x})\right],
$$



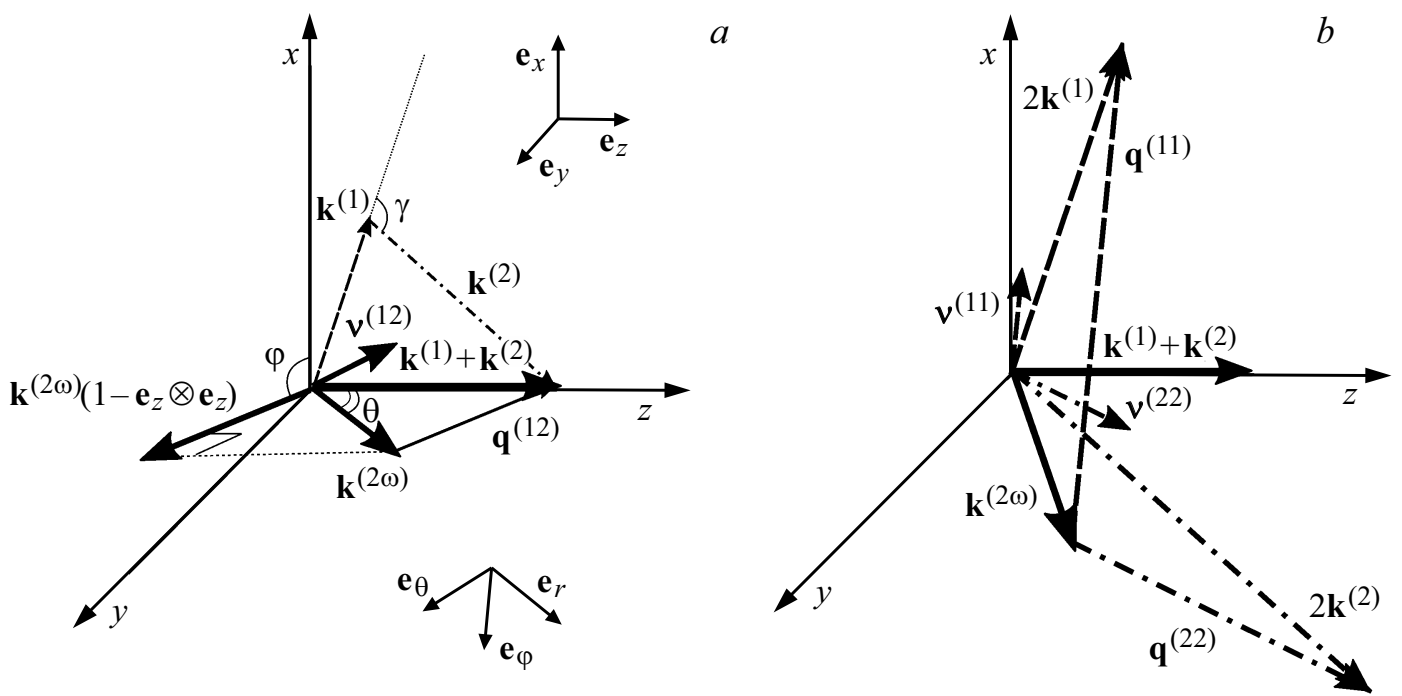

Рис. 2. Схема векторов, используемых в задаче: $(a)$ векторы, необходимые для получения $\mathbf{q}^{(12)},(b)$ векторы, необходимые для получения $\mathbf{q}^{(11)}$ и $\mathbf{q}^{(22)}$. Штриховыми линиями показаны векторы, относящиеся к первой волне. Штрихпунктирными линиями показаны векторы, относящиеся ко второй волне.

где $n_{2 \omega}-$ показатель преломления среды на частоте $2 \omega$.

Экспериментально наблюдаемой величиной является интенсивность излучения, поэтому определим радиальную компоненту вектора Умова-Пойнтинга в дальней зоне (перпендикулярные ей компоненты быстро затухают при увеличении расстояния):

$$
S_{r}^{(2 \omega)}(\mathbf{x})=S_{r}^{(2 \omega)}(r, \theta, \varphi)=\frac{c}{8 \pi} \frac{n_{2 \omega}}{\mu_{2 \omega}}\left|\mathbf{E}^{(2 \omega)}(\mathbf{x})\right|^{2} .
$$

Это выражение позволяет проводить построение трехмерных диаграмм направленности, характеризующих распределение плотности мощности генерируемого излучения второй гармоники-суммарной частоты в дальней зоне (зависимость $S_{r}^{(2 \omega)}(\theta, \varphi)$ при фиксированном $r$ ).

\section{Отношение комплексных амплитуд падающих волн}

Согласно формуле (15), пространственное распределение плотности мощности генерируемого излучения зависит не столько от значений комплексных амплитуд падающих волн, сколько от их отношения. Для удобства дальнейшего анализа введем это отношение следующим образом:

$$
\eta=\frac{E_{2}}{E_{1}}=|\eta| \exp (i \arg \eta)
$$

где параметр $\arg \eta \in(-\pi ; \pi]$ является разностью фаз падающих волн в центре частицы.

Влияние параметра $\eta$ на генерацию второй гармоники-суммарной частоты можно наблюдать на рис. 3, где приведены диаграммы направленности для отдельных слагаемых, входящих в состав $\mathbf{E}^{(2 \omega)}$ : на первых двух диаграммах направленности (рис. 3, $a, b$ ) в верхней части рис. 3 можно видеть распределение поля второй гармоники, генерируемого отдельно первой и второй волнами соответственно, а на третьей диаграмме направленности (рис. 3,c) - распределение поля, обусловленного генерацией суммарной частоты в результате взаимодействия первой и второй волн. В нижней части рис. 3 можно видеть диаграммы направленности для генерации второй гармоникисуммарной частоты, которые являются результатом суперпозиции генерируемых полей второй гармоники и суммарной частоты. Можно видеть, что при значениях $|\eta| \ll 1$ (рис. 3, $d$ ) форма диаграммы направленности для генерации второй гармоники-суммарной частоты близка к форме диаграммы направленности для генерации второй гармоники первой волной. Следовательно, в этом случае в генерацию второй гармоники суммарной частоты доминирующий вклад вносит именно генерация второй гармоники первой волной. Аналогично при $|\eta| \gg 1$ (рис. 3,f) доминирующий вклад в генерацию вносит генерация второй гармоники второй волной. Для значений $|\eta|$, близких к единице, все слагаемые в (15) имеют одинаковый порядок, поэтому генерация второй гармоники каждой из падающих волн, равно как и генерация суммарной частоты этими волнами, вносят сопоставимый вклад в результирующее поле второй гармоники-суммарной частоты (рис. 3,e).

Все эти закономерности можно объяснить, записав выражение для вектора напряженности электрического поля генерируемого излучения в следующей форме:

$$
\begin{aligned}
\mathbf{E}^{(2 \omega)}(\mathbf{x})= & 4 \pi \mu_{2 \omega} \frac{(2 \omega)^{2}}{c^{2}} \frac{\exp \left(i k_{2 \omega} r\right)}{r} d_{0} a^{2} E_{1}^{2} \\
& \times\left(1-\mathbf{e}_{r} \otimes \mathbf{e}_{r}\right) \mathbf{f}^{(2 \omega)},
\end{aligned}
$$




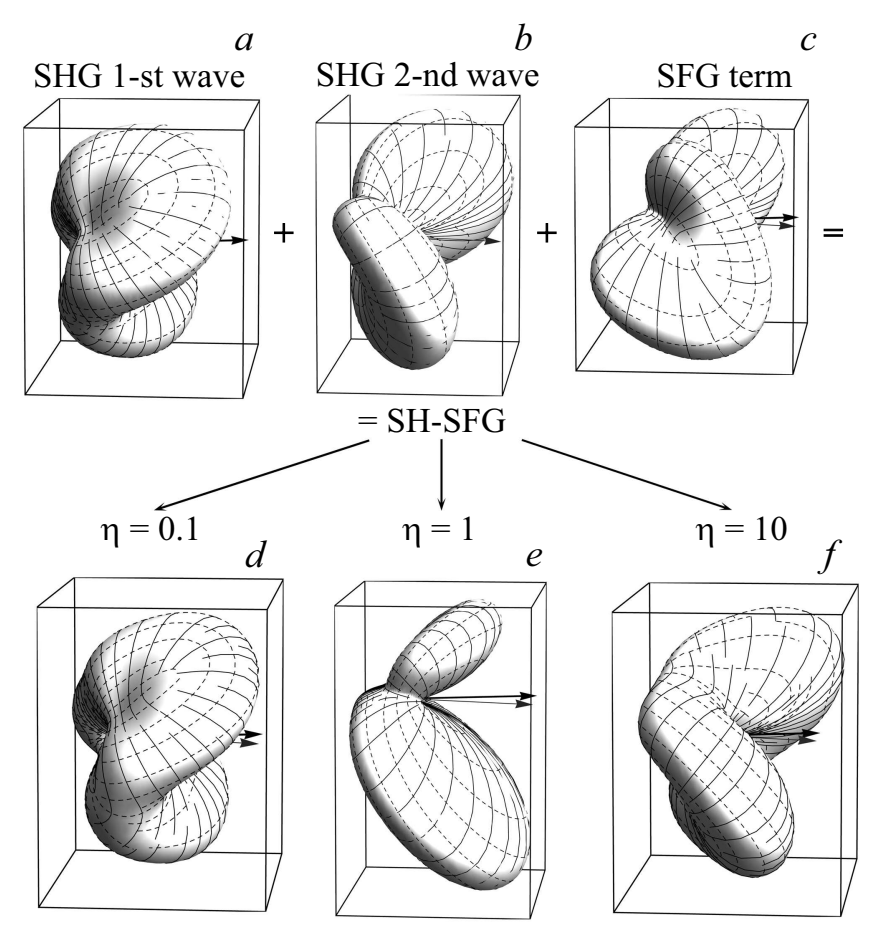

Рис. 3. Схема формирования нормированных диаграмм направленности для генерации второй гармоники-суммарной частоты. Сплошные стрелки указывают направления волновых векторов падающих волн. Утолщенные стрелки относятся к первой волне, тонкие - ко второй. Проекции больших и меньших полуосей эллипса поляризации генерируемых волн на поверхность диаграммы направленности являются касательными к сплошным и штриховым линиям на ее поверхности соответственно.

где векторная функция $\mathbf{f}^{(2 \omega)}$ характеризует пространственное распределение плотности мощности генерируемого излучения:

$$
\mathbf{f}^{(2 \omega)}=\mathbf{f}^{(11)}+2 \eta \mathbf{f}^{(12)}+\eta^{2} \mathbf{f}^{(22)}
$$

Здесь модули векторов $\mathbf{f}^{(\alpha \beta)}$, которые вычисляются по формуле (20), имеют одинаковый порядок. Из выражения (25) следует, что

$$
\begin{array}{cl}
\mathbf{f}^{(2 \omega)} \approx \mathbf{f}^{(11)}, & |\eta| \ll 1, \\
\mathbf{f}^{(2 \omega)} \approx \eta^{2} \mathbf{f}^{(22)}, & |\eta| \gg 1,
\end{array}
$$

Свойства (26) объясняют изменения формы диаграммы направленности генерируемого излучения на рис. 3, происходящие при изменении отношения амплитуд падающих волн $\eta$.

\section{Сравнение с задачей о генерации второй гармоники}

Несложно проверить, что в пределе при $|\eta| \rightarrow 0$ или $|\eta| \rightarrow \infty$ (когда плотность мощности одной падающей волны многократно превосходит плотность мощности другой волны) выражения для $\mathbf{E}^{(2 \omega)}$ в данной работе принимают следующий вид:

$$
\begin{aligned}
\mathbf{E}^{(2 \omega)}(\mathbf{x}) \rightarrow & 4 \pi \mu_{2 \omega} \frac{(2 \omega)^{2}}{c^{2}} \frac{\exp \left(i k_{2 \omega} r\right)}{r} d_{0} a^{2} E_{1}^{2} \\
& \times\left(1-\mathbf{e}_{r} \otimes \mathbf{e}_{r}\right) \mathbf{f}^{(11)}, \quad|\eta| \rightarrow 0, \\
\mathbf{E}^{(2 \omega)}(\mathbf{x}) \rightarrow & 4 \pi \mu_{2 \omega} \frac{(2 \omega)^{2}}{c^{2}} \frac{\exp \left(i k_{2 \omega} r\right)}{r} d_{0} a^{2} E_{2}^{2} \\
& \times\left(1-\mathbf{e}_{r} \otimes \mathbf{e}_{r}\right) \mathbf{f}^{(22)}, \quad|\eta| \rightarrow \infty .
\end{aligned}
$$

Эти выражения аналогичны выражению для вектора электрической напряженности $\mathbf{E}^{(2 \omega)}$ при генерации второй гармоники одной плоской волной в нелинейном сферическом слое в работе [8], т.е. в этом частном случае задача о генерации второй гармоники-суммарной частоты сводится к задаче о генерации второй гармоники.

Аналогичную закономерность можем наблюдать и при сонаправленных волновых векторах исходных волн. В этом случае получаем следующие закономерности для векторов в задаче:

$$
\begin{gathered}
\mathbf{k}^{(1)}=\mathbf{k}^{(2)}, \\
\mathbf{q}^{(11)}=\mathbf{q}^{(22)}=\mathbf{q}^{(12)}, \\
\boldsymbol{v}^{(11)}=\boldsymbol{v}^{(22)}=\boldsymbol{v}^{(12)} .
\end{gathered}
$$

Тогда выражение (15) для напряженности электрического поля принимает вид

$$
\begin{aligned}
& \mathbf{E}^{(2 \omega)}=4 \pi \mu_{2 \omega} \frac{(2 \omega)^{2}}{c^{2}} \frac{\exp \left(i k_{2 \omega} r\right)}{r} d_{0} a^{2}\left(1-\mathbf{e}_{r} \otimes \mathbf{e}_{r}\right) \\
& \times\left(E_{1}\left|\mathbf{e}^{(1)}+\eta \mathbf{e}^{(2)}\right|\right)^{2}\left[i \chi _ { 1 } ^ { ( 2 ) } \left(-j_{3}(q a) \boldsymbol{v}\left(\boldsymbol{v} \mathbf{e}^{\prime}\right)^{2}+\frac{1}{5}\left(j_{1}(q a)\right.\right.\right. \\
& \left.\left.+j_{3}(q a)\right)\left(\boldsymbol{v}\left(\mathbf{e}^{\prime} \mathbf{e}^{\prime}\right)+2 \mathbf{e}^{\prime}\left(\boldsymbol{v} \mathbf{e}^{\prime}\right)\right)\right)+i j_{1}(q a)\left(\chi_{2}^{(2)} \boldsymbol{v}\left(\mathbf{e}^{\prime} \mathbf{e}^{\prime}\right)\right. \\
& \left.\left.+2 \chi_{3}^{(2)} \mathbf{e}^{\prime}\left(\boldsymbol{v} \mathbf{e}^{\prime}\right)\right)-2 \chi_{4}^{(2)} j_{2}(q a)\left[\mathbf{e}^{\prime} \times \boldsymbol{v}\right]\left(\boldsymbol{v} \mathbf{e}^{\prime}\right)\right],
\end{aligned}
$$

где $\mathbf{e}^{\prime}$ - эквивалентный вектор поляризации, определяемый как

$$
\mathbf{e}^{\prime}=\frac{\mathbf{e}^{(1)}+\eta \mathbf{e}^{(2)}}{\left|\mathbf{e}^{(1)}+\eta \mathbf{e}^{(2)}\right|} .
$$

Выражение для вектора электрической напряженности генерируемого поля $\mathbf{E}^{(2 \omega)}$, полученное при решении задачи о генерации второй гармоники [8], полностью совпадает с выражением (29), если вектор поляризации $\mathbf{e}^{(\omega)}=\mathbf{e}^{\prime}$ и комплексная амплитуда падающей волны $E_{0}=E_{1}\left|\mathbf{e}^{(1)}+\eta \mathbf{e}^{(2)}\right|$. Следовательно, генерацию второй гармоники-суммарной частоты двумя падающими волнами при выполнении условий (28) можно рассматривать как генерацию второй гармоники одной электромагнитной волной с эквивалентным вектором поляризации.

Отметим, что эллиптичность эквивалентной волны не обязательно совпадает с эллиптичностями исходных 


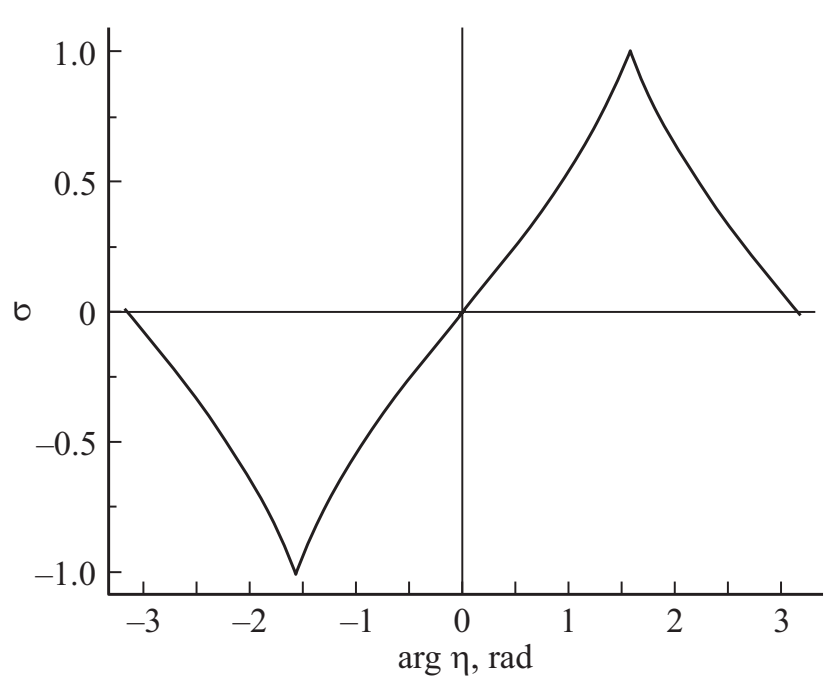

Рис. 4. Зависимость эллиптичности эквивалентной волны от разности фаз падающих волн при $|\eta|=1$.

волн. Так, например, в случае использования двух линейно поляризованных волн с перпендикулярными плоскостями поляризации $\left(\mathbf{e}^{(1)}=\mathbf{e}_{x}, \mathbf{e}^{(2)}=\mathbf{e}_{y}\right)$ эллиптичность эквивалентной волны, согласно (30), может изменяться от левоциркулярной (при $|\eta|=1, \arg \eta=-\pi / 2$ ) до правоциркулярной (при $|\eta|=1, \arg \eta=\pi / 2$ ), что можно видеть из рис. 4. Для промежуточных значений разности фаз эквивалентная волна имеет эллиптическую поляризацию.

\section{Предельные случаи}

\section{Предельные формы сферических функций Бесселя}

Особенности распределения генерируемого излучения можно проанализировать в предельных случаях: радиус частицы мал $\left(q^{(\alpha \beta)} a \ll 1\right)$ по сравнению с длиной волны падающего излучения и, наоборот, $\left(q^{(\alpha \beta)} a \gg 1\right)$. В этих двух случаях функции Бесселя в выражении (20) приближенно описываются следующими функциями:

$$
\begin{gathered}
j_{m}(z) \approx \frac{z^{m}}{(2 m+1) ! !}, \quad \text { при } \quad z \ll 1, \\
j_{m}(z) \approx \frac{\sin (z-m \pi / 2)}{z}, \quad \text { при } \quad z \gg 1 .
\end{gathered}
$$

Определим области значений переменной $z$, для которых справедливы приближения (31), при $m=1,2,3$. При малых значениях $z$ функция Бесселя имеет степенной вид, поэтому для оценки степени отклонения приближенной функции от точной можно использовать следующее условие:

$$
\left|\frac{j_{m}(z)-\frac{z^{m}}{(2 m+1) ! !}}{j_{m}(z)}\right| \leq 0.05 .
$$

Здесь число 0.05 нами выбрано в качестве максимально допустимого относительного отклонения приближенной функции от точной. Диапазоны значений переменной $z$, для которых выполняется это условие, приведены в таблице в столбце „Малые значения $z$ “.

При больших значениях аргумента $z$ сферические функции Бесселя знакопеременны и осциллируют с увеличением $z$, поэтому для оценки степени отклонения приближенной функции удобнее использовать интегральный критерий:

$$
\frac{\int_{z}^{z+2 \pi}\left(j_{m}\left(z^{\prime}\right)-\frac{\sin \left(z^{\prime}-m \pi / 2\right)}{z^{\prime}}\right)^{2} d z^{\prime}}{\int_{z}^{z+2 \pi}\left(j_{m}\left(z^{\prime}\right)\right)^{2} d z^{\prime}} \leq 0.05 .
$$

Диапазоны значений переменной $z$, для которых выполняется условие (33), приведены в таблице в столбце „Большие значения $z^{\text {“. }}$

Рассмотрим поведение функции $j_{1}(q a)$ при изменении переменной $q$ в пределах от 0 до $4 k_{\omega}$ и различных значениях параметра $a$ (рис. 5). Выбран именно такой диапазон изменения переменной $q$, так как модуль вектора рассеяния $\mathbf{q}^{(\alpha \beta)}$ принимает наименьшее значение для сонаправленных векторов $\mathbf{k}^{(\alpha)}, \mathbf{k}^{(\beta)}$ и $\mathbf{k}^{(2 \omega)}$ (для малой дисперсии $q^{(\alpha \beta)} \approx 0$ ) и наибольшее для сонаправленных векторов $\mathbf{k}^{(\alpha)}, \mathbf{k}^{(\beta)}$ и противоположно направленного вектора $\mathbf{k}^{(2 \omega)}\left(q^{(\alpha \beta)} \approx 4 k_{\omega}\right)$. На рис. 5, $a$ изображен график зависимости $j_{1}(q a)$ от $q / k_{\omega}$ при $a=10 / k_{\omega}$. В левой части графика закрашена область, в которой выполняется условие $0 \leq q a \leq 0.696$, т.е. справедливо приближение $j_{1}(q a)=q a / 3$ (таблица). В правой части графика закрашена область, где выполняется условие $q a \geq 4.279$, т.е. справедливо приближение $j_{1}(q a)=-\cos (q a) /(q a)$. По центру находится незакрашенная область, где для сферической функции Бесселя первого порядка указанные выше приближения нельзя применять.

На рис. $5, b$, где $a=1 / k_{\omega}$, закрашена только область в левой части графика, где справедливо приближение для малых значений аргумента сферической функции Бесселя, а во всей правой незакрашенной части графика для расчетов необходимо использовать функцию $j_{1}(q a)$. На рис. $5, c$, где $a=0.1 / k_{\omega}$, вся площадь под графиком закрашена, т.е. для любых значений $q$ для функции $j_{1}(q a)$ можно использовать приближение малого аргумента.

Из графиков на рис. 5 можно видеть, что при любых значениях параметра $a$ приближение сферической функции Бесселя $j_{1}(q a)$ для малого аргумента может быть использовано в некотором диапазоне значений $q$. Причем ширина диапазона применимости растет с уменьшением значения параметра $a$ и при некотором значении $а$ совпадает с областью построения. Как следствие, приближенная формула функций Бесселя может быть использована для всех возможных направлений наблюдения $\theta, \varphi$. Область применимости приближения 

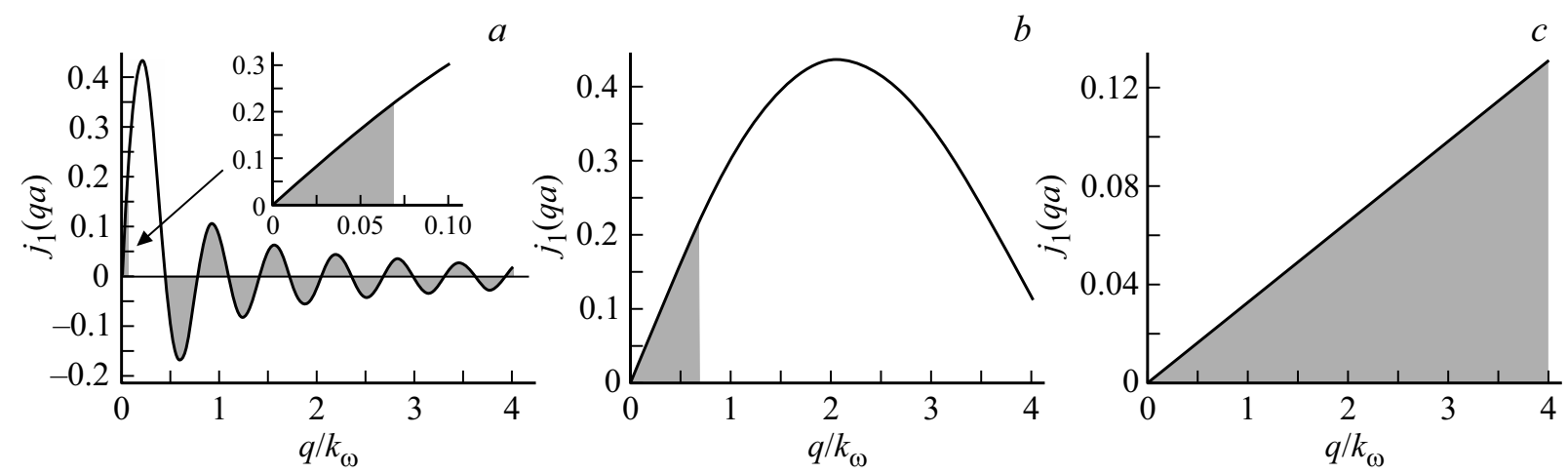

Рис. 5. Зависимость сферической функции Бесселя первого порядка с аргументом $q a$ от величины $q / k_{\omega}$ для различных значений радиуса сферического слоя: $a=10 / k_{\omega}(a), 1 / k_{\omega}(b), 0.1 / k_{\omega}(c)$.

Области применимости приближений для предельных случаев

\begin{tabular}{c|c|c|c|c}
\hline \multirow{2}{*}{ Функция Бесселя } & \multicolumn{2}{|c|}{ Малые значения $z$} & \multicolumn{2}{|c}{ Большие значения $z$} \\
\cline { 1 - 4 } & Приближение & Условие & Приближение & Условие \\
\hline$j_{1}(z)$ & $z / 3$ & $0 \leq z \leq 0.696$ & $-\cos (z) / z$ & $z \geq 4.279$ \\
$j_{2}(z)$ & $z^{2} / 15$ & $0 \leq z \leq 0.824$ & $-\sin (z) / z$ & $z \geq 12.344$ \\
$j_{3}(z)$ & $z^{3} / 105$ & $0 \leq z \leq 0.935$ & $\cos (z) / z$ & $z \geq 26.208$
\end{tabular}

для больших аргументов присутствует на графике только при больших значениях параметра $a$, а диапазон значений $q$, при которых оно применимо, увеличивается с ростом значения параметра $a$. Отметим, что глобальный максимум сферической функции Бесселя первого порядка находится в диапазоне неприменимости рассмотренных нами приближений, если этот диапазон входит в область построения. Очевидно, что с увеличением параметра $a$ значение $q$, при котором наблюдается глобальный максимум $j_{1}(q a)$, смещается в сторону нуля. Несложно проверить, что аналогичные закономерности сохраняются и для сферических функций Бесселя старших порядков $j_{m}(q a)(m=2,3)$, но границы диапазонов применимости приближений изменяются в соответствии с условиями в таблице.

\section{Предельные формы решения}

В случае малого радиуса частицы $\left(q^{(\alpha \beta)} a \ll 1\right)$ формулы для $\mathbf{f}^{(\alpha \beta)}$ (в пренебрежении слагаемыми выше второго порядка) могут быть представлены в виде

$$
\begin{aligned}
& \mathbf{f}^{(\alpha \beta)} \approx i \frac{q^{(\alpha \beta)} a}{15} \chi_{1}^{(2)}\left(\boldsymbol{v}^{(\alpha \beta)}\left(\mathbf{e}^{(\alpha)} \mathbf{e}^{(\beta)}\right)+\mathbf{e}^{(\beta)}\left(\boldsymbol{v}^{(\alpha \beta)} \mathbf{e}^{(\alpha)}\right)\right. \\
& \left.+\mathbf{e}^{(\alpha)}\left(\boldsymbol{v}^{(\alpha \beta)} \mathbf{e}^{(\beta)}\right)\right)+i \frac{q^{(\alpha \beta)} a}{3}\left(\chi_{2}^{(2)} \mathbf{v}^{(\alpha \beta)}\left(\mathbf{e}^{(\alpha)} \mathbf{e}^{(\beta)}\right)\right. \\
& \left.+\chi_{3}^{(2)} \mathbf{e}^{(\beta)}\left(\boldsymbol{v}^{(\alpha \beta)} \mathbf{e}^{(\alpha)}\right)+\chi_{3}^{(2)} \mathbf{e}^{(\alpha)}\left(\boldsymbol{v}^{(\alpha \beta)} \mathbf{e}^{(\beta)}\right)\right)-\frac{\left(q^{(\alpha \beta)} a\right)^{2}}{15} \\
& \times \chi_{4}^{(2)}\left(\left[\mathbf{e}^{(\alpha)} \times \boldsymbol{v}^{(\alpha \beta)}\right]\left(\boldsymbol{v}^{(\alpha \beta)} \mathbf{e}^{(\beta)}\right)+\left[\mathbf{e}^{(\beta)} \times \boldsymbol{v}^{(\alpha \beta)}\right]\left(\boldsymbol{v}^{(\alpha \beta)} \mathbf{e}^{(\alpha)}\right)\right) .
\end{aligned}
$$

Используя рассуждения предыдущего пункта, можно сделать вывод, что выражение для $\mathbf{f}^{(\alpha \beta)}$ на основе приближения функции Бесселя для малого аргумента может быть применено не только при малых значениях радиуса сферического слоя $a$, но и при других значениях $a$, если $q^{(\alpha \beta)}$ достаточно мало. Это условие выполняется при генерации в направлениях, для которых вектор $\mathbf{k}^{(2 \omega)}$, характеризующий направление наблюдения, близок к вектору $\mathbf{k}^{(\alpha)}+\mathbf{k}^{(\beta)}$.

Согласно (34), для малых частиц наибольший вклад в генерируемое излучение вносят некиральные слагаемые, для них $S_{r}^{(2 \omega)} \sim a^{6}$, в то время как для исключительно кирального слоя $\left(\chi_{1-3}^{(2)}=0, \chi_{4}^{(2)} \neq 0\right) S_{r}^{(2 \omega)} \sim a^{8}$. Такой же порядок зависимости от размеров частицы имеют слагаемые, обусловленные киральными и некиральными компонентами в работе [8], посвященной генерации второй гармоники в нелинейном сферическом слое.

Для генерации суммарной частоты электромагнитными волнами с разными частотами [10] зависимость 
плотности мощности генерируемого излучения от радиуса имеет порядок $a^{4}$, обусловленный доминирующими киральными компонентами в выражении для $\mathbf{f}^{(12)}$ из работы [10]. При переходе от этого случая к генерации второй гармоники-суммарной частоты доминирующие киральные слагаемые обращаются в ноль из-за необходимости выполнения свойств симметрии относительно перестановки индексов. Отметим также, что некиральные компоненты в [10] при этом симметричны относительно перестановки падающих волн, а порядок зависимости генерируемой плотности мощности $S_{r}^{(2 \omega)} \sim a^{6}$.

При больших значениях $q^{(\alpha \beta)} a\left(q^{(\alpha \beta)} a \gg 1\right)$ выражения для векторов $\mathbf{f}^{(\alpha \beta)}$ имеют вид

$$
\begin{aligned}
& \mathbf{f}^{(\alpha \beta)} \approx-i \frac{\cos \left(q^{(\alpha \beta)} a\right)}{q^{(\alpha \beta)} a}\left[\chi_{1}^{(2)} \mathbf{v}^{(\alpha \beta)}\left(\boldsymbol{v}^{(\alpha \beta)} \mathbf{e}^{(\alpha)}\right)\left(\boldsymbol{v}^{(\alpha \beta)} \mathbf{e}^{(\beta)}\right)\right. \\
& +\chi_{2}^{(2)} \boldsymbol{v}^{(\alpha \beta)}\left(\mathbf{e}^{(\alpha)} \mathbf{e}^{(\beta)}\right)+\chi_{3}^{(2)} \mathbf{e}^{(\beta)}\left(\boldsymbol{v}^{(\alpha \beta)} \mathbf{e}^{(\alpha)}\right) \\
& \left.+\chi_{3}^{(2)} \mathbf{e}^{(\alpha)}\left(\boldsymbol{v}^{(\alpha \beta)} \mathbf{e}^{(\beta)}\right)\right]+\chi_{4}^{(2)} \frac{\sin \left(q^{(\alpha \beta)} a\right)}{q^{(\alpha \beta)} a}\left(\left[\mathbf{e}^{(\alpha)} \times \boldsymbol{v}^{(\alpha \beta)}\right]\right. \\
& \left.\times\left(\boldsymbol{v}^{(\alpha \beta)} \mathbf{e}^{(\beta)}\right)+\left[\mathbf{e}^{(\beta)} \times \boldsymbol{v}^{(\alpha \beta)}\right]\left(\boldsymbol{v}^{(\alpha \beta)} \mathbf{e}^{(\alpha)}\right)\right) .
\end{aligned}
$$

Принимая во внимание рассуждения предыдущего пункта, можно сказать, что приближение функции Бесселя для большого аргумента даже при больших радиусах нелинейного слоя $a$ применимо только тогда, когда параметр $q^{(\alpha \beta)}$ превышает некоторое пороговое значение (которое можно найти, пользуясь таблицей), т.е. при достаточном отклонении вектора $\mathbf{k}^{(2 \omega)}$ от вектора $\mathbf{k}^{(\alpha)}+\mathbf{k}^{(\beta)}$. При чуть меньшем отклонении для $\mathbf{f}^{(\alpha \beta)}$ применима формула (20), а при совсем незначительном отклонении $\mathbf{k}^{(2 \omega)}$ от $\mathbf{k}^{(\alpha)}+\mathbf{k}^{(\beta)}$ даже при очень больших значениях радиуса $a$ для $\mathbf{f}^{(\alpha \beta)}$ справедливо приближение (34).

Согласно условиям (1), в данном случае, чтобы не выйти за рамки приближенной модели, разность между показателями преломления среды и частицы на частотах $\omega$ и $2 \omega$ должна быть крайне мала. При большом радиусе частицы диаграммы направленности будут состоять из многочисленных лепестков, что объясняется наличием знакопеременных функций $\cos \left(q^{(\alpha \beta)} a\right) / q^{(\alpha \beta)} a$ и $\sin \left(q^{(\alpha \beta)} a\right) / q^{(\alpha \beta)} a$, имеющих множество локальных максимумов и минимумов.

\section{Заключение}

В настоящей работе в рамках модели Рэлея-Ганса-Дебая получено аналитическое решение задачи о генерации второй гармоники-суммарной частоты в тонком сферическом слое, обладающем нелинейными свойствами, при облучении его двумя когерентными плоскими эллиптически поляризованными электромагнитными волнами.

Показано, что отношение комплексных амплитуд падающих волн определяет вклад генерации второй гар- моники каждой из волн в отдельности и генерации суммарной частоты в пространственное распределение поля второй гармоники-суммарной частоты. В предельных случаях, когда амплитуда одной падающей волны значительно больше другой, доказано совпадение выражений для вектора напряженности генерируемого поля в задаче о генерации второй гармники-суммарной частоты с аналогичными выражениями в задаче о генерации второй гармоники от одного источника [8]. Для случая сонаправленных волновых векторов падающих волн также показано совпадение выражений, характеризующих генерируемое излучение удвоенной частоты, в работе [8] и в настоящей работе.

Найдены предельные формы функций, характеризующих распределение генерируемого излучения, и области их применимости. Анализ предельных форм решения показал, что для частиц, размер которых много меньше длины волны падающего излучения, генерация обусловлена в большей степени некиральными слагаемыми. При этом объяснены сходства и различия между явлениями генерации второй гармоники и генерации суммарной частоты для данного предельного случая. Предсказано распределение генерируемого излучения в виде большого количества лепестков, если частицы имеют большой размер по сравнению с длиной волны возбуждающего излучения.

\section{Финансирование работы}

Работа выполнена при финансовой поддержке Белорусского республиканского фонда фундаментальных исследований (грант Ф20М-011).

\section{Конфликт интересов}

Авторы заявляют, что у них нет конфликта интересов.

\section{Список литературы}

[1] Wang H., Yan E.C.Y., Borguet E., Eisenthal K.B. // Chem. Phys. Lett. 1996. V. 259. N 1-2. P. 15. doi 10.1016/0009-2614(96)00707-5

[2] Yang N., Angerer W.E., Yodh A.G. // Phys. Rev. Lett. 2001. V. 87. N 10. P. 103902. doi 10.1103/PhysRevLett.87.103902

[3] Шамына А.А., Капшай В.Н. // Опт. и спектр. 2018. T. 124. № 1. C. 105. doi 10.21883/OS.2018.01.45366.176-17; Shamyna A.A., Kapshai V.N. // Opt. Spectrosc. 2018. V. 124. N 1. P. 103. doi 10.1134/S0030400X18010198

[4] de Beer A.G.F., Roke S. // Phys. Rev. B. 2007. V. 75. N 24. P. 245438. doi 10.1103/PhysRevB.75.245438

[5] Viarbitskaya S., Kapshai V., P. vander Meulen, Hansson T. // Phys. Rev. A. 2010. V. 81. N 5. P. 053850. doi 10.1103/PhysRevA.81.053850

[6] Martorell J., Vilaseca R., Corbalan R. // Phys. Rev. A. 1997. V. 55. N 6. P. 4520. doi 10.1103/PhysRevA.55

[7] Dadap J.I., Shan J., Heinz T.F. // JOSA. B. 2004. V. 21. N 7. P. 1328. doi 10.1364/JOSAB.21.001328 
[8] Капшай В.Н., Шамына А.А. // Опт. и спектр. 2017. T. 123. № 3. C. 416. doi 10.7868/S003040341709015X; Kapshai V.N., Shamyna A.A. // Opt. Spectrosc. 2017. V. 123. N 3. P. 440. doi 10.1134/S0030400X17090144

[9] Freund I., Deutsch M., Sprecher A. // Biophys. J. 1986. V. 50. N 4. P. 693. doi 10.1016/S0006-3495(86)83510-X

[10] Капшай В.Н., Шамына А.А. // Опт. и спектр. 2018. T. 124. № 6. C. 795. doi 10.21883/OS.2018.06.46083.55-18; Kapshai V.N., Shamyna A.A. // Opt. Spectrosc. 2018. V. 124.

N 6. P. 826. doi 10.1134/S0030400X18060115 